\title{
Designing a Heterogeneous Sensor Tier for the EMULSION IoT Platform
}

\author{
IVAN GANCHEV \\ Department of Computer Systems, \\ University of Plovdiv "Paisii Hilendarski", \\ 24 Tsar Assen St., Plovdiv 4000, \\ BULGARIA. \\ $\&$ \\ Institute of Mathematics and Informatics, \\ Bulgarian Academy of Sciences, \\ Akad. G. Bonchev St., Block 8, Sofia 1113, BULGARIA. \\ $\&$ \\ Telecommunications Research Centre (TRC), \\ University of Limerick, \\ Plassey, National Technological Park, Co. Limerick, \\ IRELAND. \\ https://orcid.org/0000-0003-0535-7087

\section{ZHANLIN JI} \\ College of Artificial Intelligence, \\ North China University of Science and Technology, \\ Caofeidian, Tangshan City, \\ CHINA. \\ https://orcid.org/0000-0003-3527-3773
}

\begin{abstract}
The design of a heterogeneous sensor tier for the generic, multi-service, cloud-based, IoT operational platform EMULSION is presented in this paper, along with typical hardware examples and deployment schemas involving different types of sensor sets and monitoring stations for detecting and notifying about the changes occurring in the physical world. Aided by the communication tier, the sensor tier deals also with issues of heterogeneity related to the integration of different IoT things, objects, devices, etc., into EMULSION. The elaborated multi-tiered structure of the platform, allowing much flexibility of the service provisioning along with easy scalability and expandability, is presented as well.
\end{abstract}

Keywords: - IoT platform, horizontal-type, heterogeneity, sensor tier, remote sensing.

Received: March 21, 2021. Revised: January 12, 2022. Accepted: January 27, 2022. Published: February $18,2022$.

\section{Introduction}

In 2021, the Internet of Things (IoT) market turned to 12.3 billion connected IoT devices and $\$ 160$ billion in IoT enterprise spending [1]. The same source suggests that the combination of artificial intelligence (AI) and IoT (i.e., the so-called AIoT) will increase the global industrial AIoT market to $\$ 102$ billion by 2026 . There are lots of interesting (and big!) IoT projects currently underway and some of the more promising ones are identified also in [1]. For instance, in January 2021, China announced its 3-year plan for creating 30 fully- connected fifth-generation (5G) factories based on industrial IoT (IIoT) platforms, e.g., with an ability to perform quality control using high-definition cameras supported by AI. Recently, PTC and Microsoft have proposed the term "industrial metaverse" for describing mixed and augmented reality scenarios for IIoT-based product development and manufacturing. IoT is widely embraced also by the city authorities around the world as to establish the so-called 'smart cities'. For instance, the last-year-published IoT strategy for the city of New York (NY) builds on already existing 
IoT initiatives in NY (e.g., 23,000 connected public transport vehicles, myriad of smart connected speed cameras, 800,000 connected water meters, multiple air quality index (AQI) monitoring stations, and bike counters), and includes plans for further training, funding, consultancy and coordination of deployment of the supporting communication networks (e.g., 5G cellular, Narrow-Band IoT (NBIoT), LOng-RAnge Wide Area Network (LoraWAN), e.g., for connecting temperature and humidity sensors in an attempt to analyze the effect of various city-wide running initiatives on NY citizens. IoT is now moving even to the satellite domain in the form of a satellite IoT, aimed at providing ubiquitous (nano)satellite connectivity for IoT devices, [1].

However, in general, IoT still lacks complete solutions to multiple problems associated with the integration of heterogeneous things, objects, devices, etc., accessible via heterogeneous communication networks on one hand, and achieving full interoperability of the involved hardware and software components on the other hand, in order to achieve seamless (and more secure!) provision of IoT services to the involved consumers. For this, a well-designed and stablyoperating IoT platform is needed at first place, for providing an efficient, flexible, reliable, and secure solution that meets today's system architectural and business requirements [2].

The existing IoT platforms are mostly of a vertical type, meaning they are focused on the service provision within a single IoT sector, e.g., IIoT, energy production and distribution, supply chain, autonomous cars, smart health, smart environment control, smart agriculture, smart homes, etc. By utilizing such platforms, IoT providers can operate in a vertical manner, involving separate applications and services, separate network connections, and separate things, objects, and devices for the provision of a particular service, as shown in Fig. 1. The result of applying this vertical approach was the creation of the 'Internet of Silos' instead of real IoT, with all corresponding drawbacks, such as further fragmentation of the IoT sectors, difficult interoperability and integration, increased OPerational EXpenditure (OPEX), and obstacles to achieving sufficient scaling and openness to new IoT services. As a result, there were 613 IoT platform vendors existing by the end of 2021 (almost the same number as in 2019, i.e., 620, but up from 450 in 2017). However, the platforms of the top 10 providers (i.e., Microsoft's Azure IoT Hub, AWS IoT Core, Google Cloud, and few others) have
$65 \%$ market share (up from 58\% in 2019 and $44 \%$ in 2016) [3].

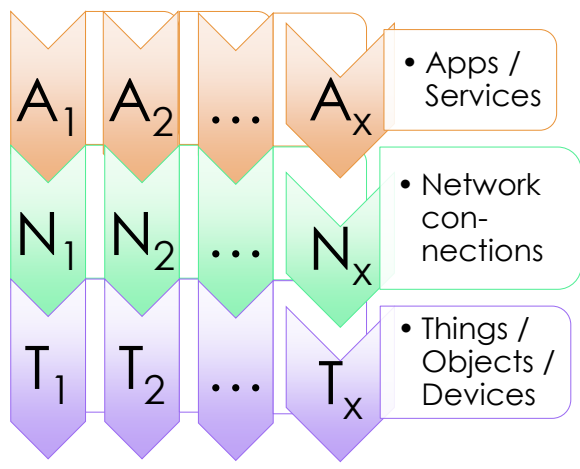

Fig. 1. The vertical approach for the creation of IoT platforms.

The new horizontal approach for building IoT platforms, shown in Fig. 2, overcomes many of the drawbacks of the vertical approach, by allowing a provider to bring only a horizontal slice in the delivery of IoT services. This horizontal principle meets better today's expectations for efficiency and effectiveness, flexibility and scalability, easy adjustment and openness to new IoT use cases and scenarios, and multi-purpose use of the built platforms. In addition, it ensures simplification of the IoT environment by removing the duplicate solutions and ensuring inter-technology operation, opening of new IoT business opportunities, and efficient Operation, Administration, and Management (OAM) of the whole IoT ecosystem throughout the lifespan.

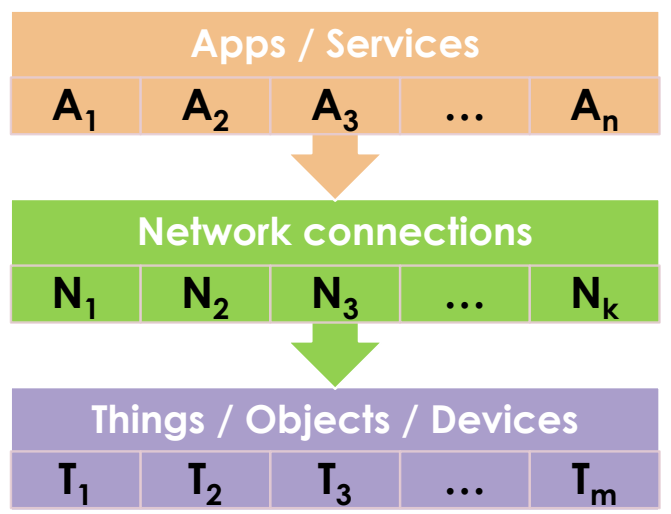

Fig. 2. The horizontal approach for the creation of IoT platforms.

Designing a reliable, robust, and low-cost IoT platform of a combined (hardware and software) type is a difficult task. With millions of instances of open-source hardware and software on the GitHub ${ }^{1}$,

${ }^{1}$ https://github.com/ 
selecting the proper hardware modules and appropriate software code is a big challenge in numerous technical routes.

The design aspects of a horizontal-type, generic, cloud-based, IoT operational platform, called EMULSION [4], are summarized in this paper, with a particular focus on its heterogeneous sensor tier, which (aided by the communication tier) solves the associated problems of integration of different-type IoT things, objects, devices, etc., into the platform. Other characteristics of EMULSION include flexibility, scalability, easy expandability, openness, and multi-service provisioning.

\section{EMULSION IoT Platform}

EMULSION is being built by means of low-cost electronic modules and open-source software components, by utilizing the multi-tier IoT architecture shown in Fig. 3 and Fig. 4. Multiple sensors (S), location trackers (T) [5], and monitoring stations (MS) are deployed in the sensor tier for detecting and recording of changes occuring in the physical world. The collected data are sent to the information center(s), positioned in the cloud tier, by means of Data/Remote Transfer Units (D/RTUs) [6], furnished with wireless communication modules of different type. Smart communication gateways facilitate communication between heterogeneous IoT things, objects, and devices, accessible directly or indirectly through Wireless Sensor Networks (WSNs). After analyzing the data collected from all respective D/RTUs and WSNs, the information center(s) send back the corresponding decisions and recommendations to the actuators (A), controllers (C), and guards $(\mathrm{G})$, located in the sensor tier, for enforcing the right OAM measures and realizing the desired changes in the physical world.


Fig. 3. The IoT multi-tier architecture of EMULSION.

EMULSION consists of the following seven tiers, as shown in Fig. 4:

1) Sensor tier - involving a variety of sensor sets and monitoring stations for detecting and notifying about the changes in the physical world.

2) Communication tier - used for ensuring seamless and transparent heterogeneous internetworking by means of smart communication gateways that 'intelligently' operate between different communication networks, such as: (i) Low-Power Wide Area Networks (LPWANs), e.g., LoRa and Sigfox; (ii) Wi-Fi networks (the IEEE
802.11 family of Wireless Local Area Networks, WLANs); (iii) Wireless Personal Area Networks (WPANs), e.g., based on Bluetooth Low Energy (BLE); (iv) cellular networks, including all their current and future generations $(2 \mathrm{G} \div 6 \mathrm{G})$, but also all LPWANs based on the Third Generation Partnership Project (3GPP) standards, such as Long Term Evolution for Machines (LTE-M) - Cat-M1 and Narrow-Band Internet of Things (NB-IoT), etc.

Each smart communication gateway may also contain a fog node for performing initial analysis of data, collected from the sensor tier, and for 
generating knowledge as close as possible to the data source. The fog nodes can also help set up and configure secure communication sessions between the sensor tier and cloud tier [7]. The overall goal of the communication tier is to allow simultaneous communication of up to $1,000,000$ heterogeneous IoT devices within a single EMULSION cluster.

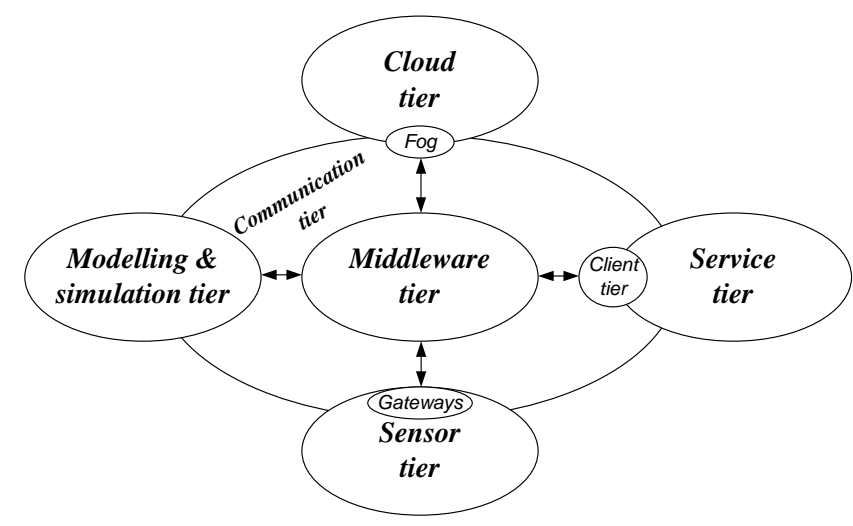

Fig. 4. The 7-tier structure of EMULSION.

3) Modelling \& simulation tier - used for modeling of cyber-physical-social (CPS) objects and IoT services (and their attributes and temporal/spatial/event characteristics). The Generalized Nets modelling [8] [9] is used for optimizing the queuing disciplines applied to the incoming service requests. Moreover, this tier is used also to simulate the actual provision of IoT services as to figure out (by solving complex optimization tasks) the optimal configuration of the platform for each particular deployment case.

4) Client tier - used for facilitating the consumers' access to services of the platform, in compliance with the Always Best Connected and best Served (ABC\&S) communication paradigm [10].

5) Service tier - used for supplying regular IoT services and supplementary services for finding and recommending to consumers the 'best' instances of the regular IoT services.

A sample IoT service, which is being developed for the 'smart environment control' IoT sector, utilizes live AQI data provided by monitoring stations, located in the sensor tier, along with AQI forecasting information from the service tier, for advanced proactive planning of travel routes through polluted areas with minimum health risk to consumers. More details about this service are provided in the next section.

6) Middleware tier - operating as a medium for ensuring the required interoperability between the other tiers [7].
7) Cloud tier - used for providing the required data analysis and for generating respective commands, decisions, and recommendations to the sensor tier.

\section{Sensor Tier of EMULSION}

The first group of IoT devices, utilized by this tier, includes a variety of sensor sets and monitoring stations for detecting and notifying about the changes occurring in the physical world. In the 'smart environment control' IoT sector, the focus of this tier is on the AQI monitoring for the purposes of daily live reporting (and forecasting) of AQI values in the target area(s).

The multi-parametric AQI monitoring stations used include both commercial ones (Fig. 5) and selfelaborated ones (Fig. 6 and Fig. 7). These are used to capture and save (and possibly also analyse) the data about the $\mathrm{PM}_{1}, \mathrm{PM}_{2.5}, \mathrm{PM}_{10}, \mathrm{CO}_{2}, \mathrm{SO}_{2}, \mathrm{NO}_{2}$, $\mathrm{O}_{3}$, temperature, humidity, pressure, and luminosity values of the atmospheric environment in the area(s) of interest. The AQI data obtained by these monitoring stations are used for elaborating short/long-term forecasting techniques with respect to changes in the atmospheric environment in the area(s) of interest. The produced forecasts are utilized for the smart pro-active route planning service that is being developed for EMULSION.

The AQI monitoring stations are located at geogrid points in the area(s) of interest (Fig. 8), and will be used for conducting field-trial experimental testing, validation, and verification of the pilot $A Q I$ monitoring system, which is being built, based on the EMULSION IoT platform (Fig. 9 and Fig. 10). The grid consists (predominantly) of fixed AQI monitoring stations. In addition, mobile AQI monitoring stations, mounted on participating vehicles, are also used in order to obtain the actual AQI values on the roads of particular interest. For instance, as shown in Fig. 8, the use of such mobile AQI monitoring station would facilitate the determination of route 3 as the 'healthiest' one, instead of route 4 , which otherwise would have been selected in that capacity. The mobile AQI monitoring stations are being developed by utilizing the Raspberry Pi 4 technology. Each such station includes a variety of sensors for measuring different components of the AQI value, such as a SDS011 Nova $\mathrm{PM}_{2.5} / \mathrm{PM}_{10}$ sensor, a $\mathrm{ZE} 25-\mathrm{O}_{3}$ ozone sensor, a ZE03 $\mathrm{SO}_{2}$ sensor, a ZE03 $\mathrm{NO}_{2}$ sensor, a ZE03 CO sensor, etc. 


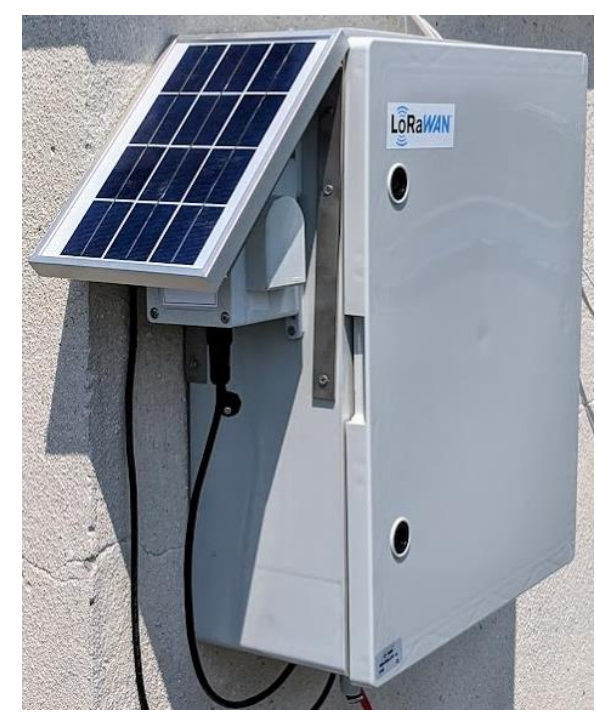

Fig. 5. A commercial AQI monitoring station of the "Paisii Hilendarski" University of Plovdiv, utilized by

EMULSION (https://smartcity.shieldo.space/).

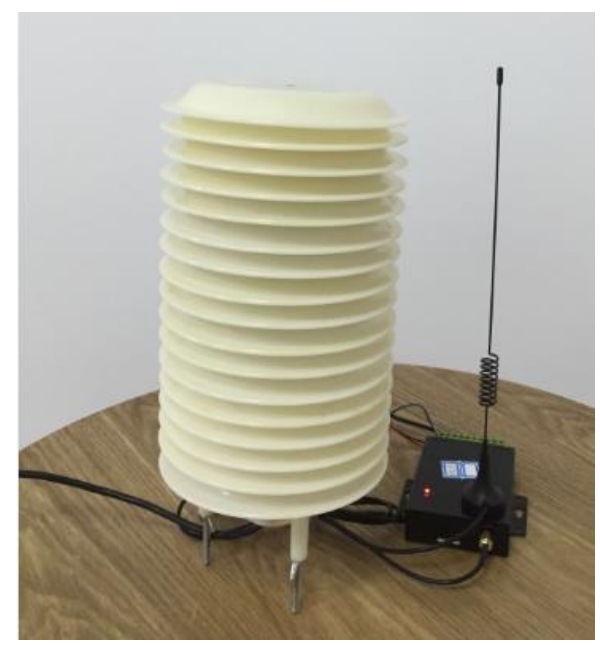

Fig. 6. A sample self-elaborated AQI monitoring station, utilized by EMULSION.

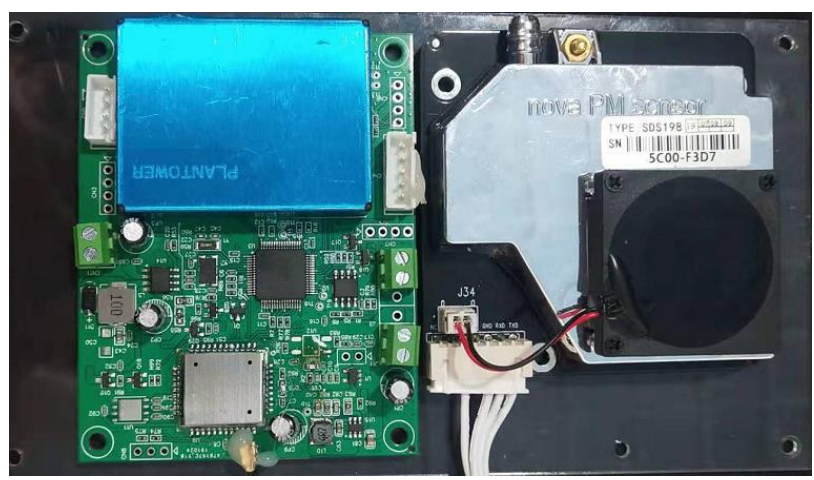

Fig. 7. A sample hardware of a self-elaborated AQI monitoring station, utilized by EMULSION.

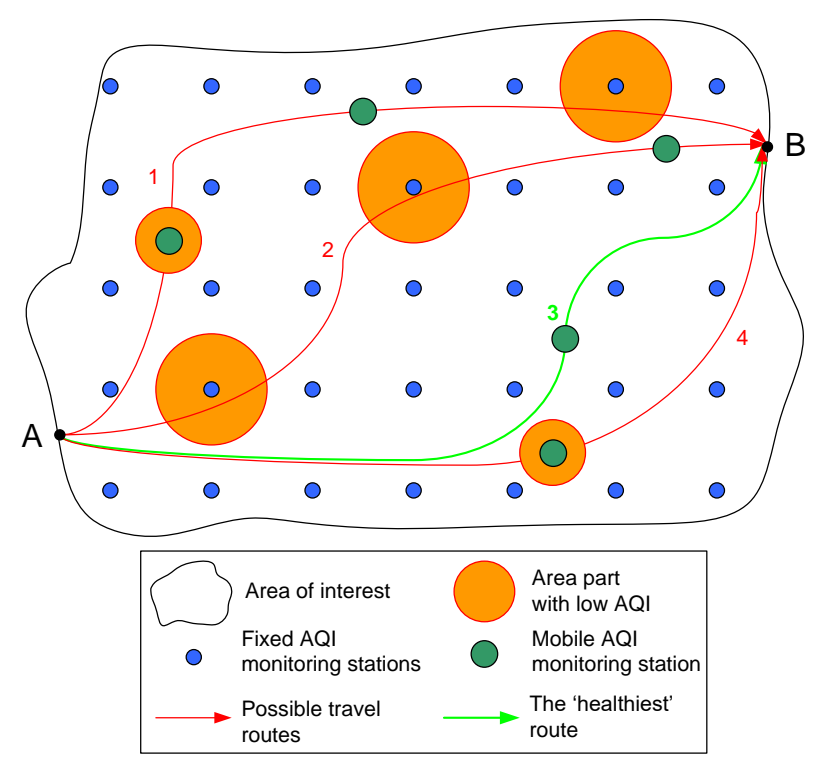

Fig. 8. An illustration of using a geo-grid of AQI monitoring stations for determining the 'healthiest' route of a consumer for moving through an area.

The D/RTUs, utilized to connect sensors to smart communication gateways (c.f. Fig. 3), consist of the following three main modules:

\section{- Power-supply module}

This module is important for guaranteeing the stand-alone functioning of battery-powered D/RTUs. Consecutively, its design is vital for the successful operation of the entire IoT platform. The sample power-supply module, developed for EMULSION, has an $5 \mathrm{~V}-18 \mathrm{~V}$ input voltage range and provides an output of $3.3 \mathrm{~V}$ or $4.2 \mathrm{~V}$ for the wireless communication module and $3.3 \mathrm{~V}$ for the MCU and the peripheral circuit (Fig. 11).

\section{- MCU module}

The MCU design, allowing remote system update and upgrade, is very important as this is a key requisite for any IoT platform. For EMULSION, the most widely used type is the Arm Cortex-M3 STM32F103RE, utilized in the design of single-chip systems. It has a $512-\mathrm{kB}$ flash memory, a $72-\mathrm{MHz} \mathrm{CPU}$, and can work in a temperature range of $-40^{\circ} \mathrm{C}$ to $+105^{\circ} \mathrm{C}$. To communicate with sensors, it provides three 12-bit ADCs, two I2Cs, 11 timers, three SPIs, and five USARTs. To enable rapid development, the core board was designed with Winbond W25Q32JVTCIQ flash, and 8M and $32 \mathrm{M}$ crystal oscillators. The layout of the developed printed circuit board (PCB) is shown in Fig. 12. 


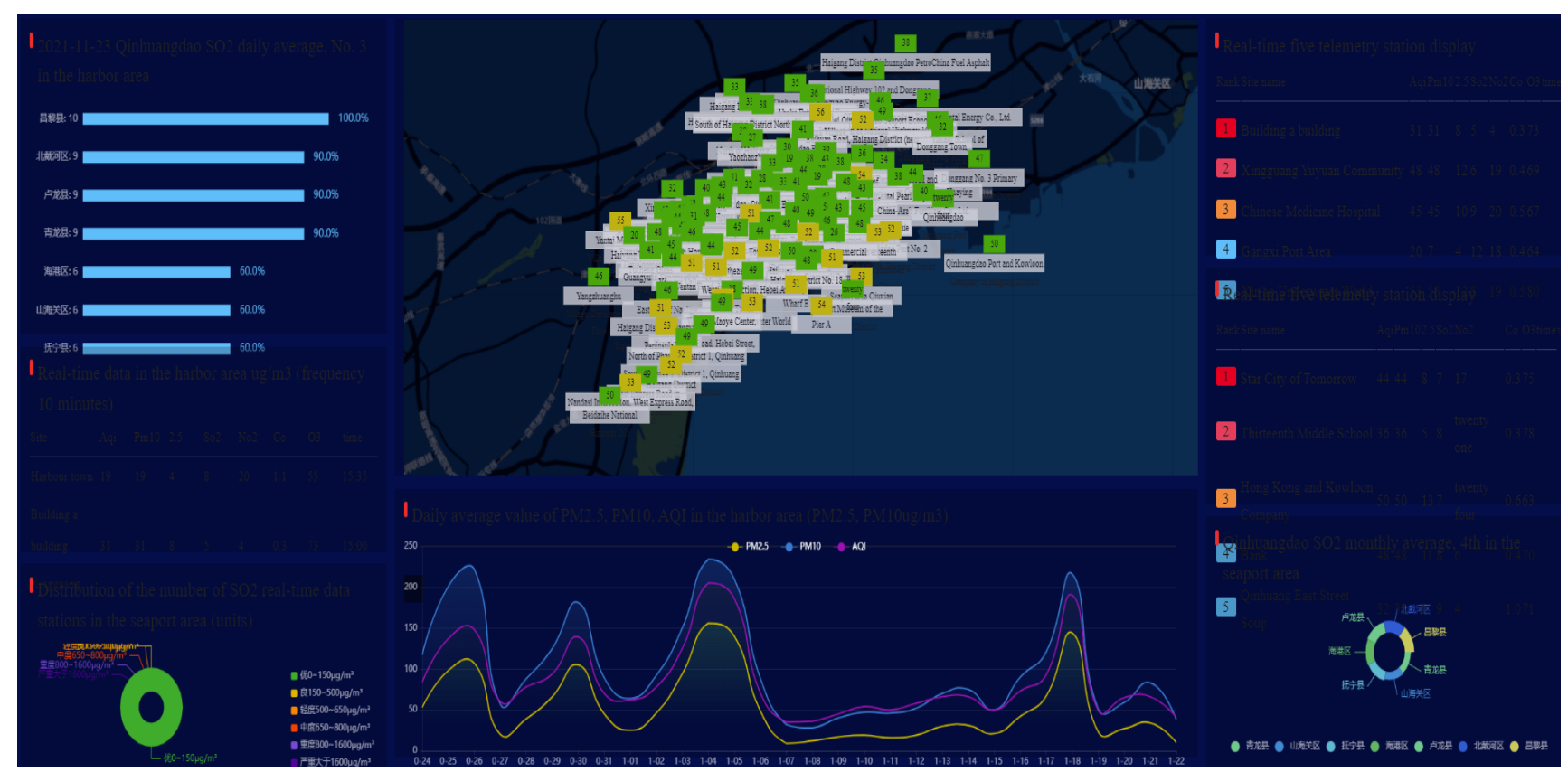

Fig. 9. The pilot AQI monitoring system: the prototype dashboard.

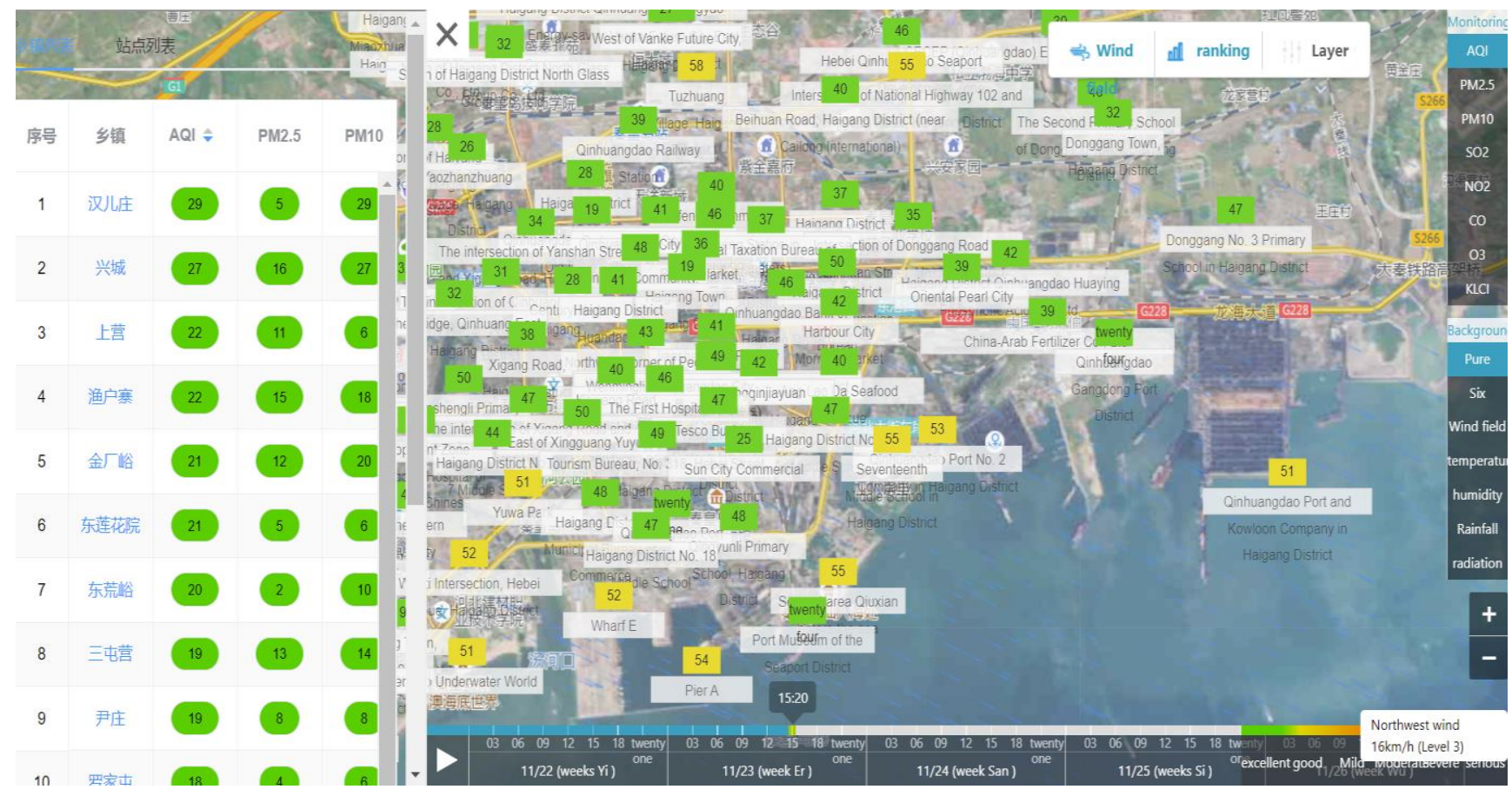

Fig. 10. The pilot AQI monitoring system: the website view. 


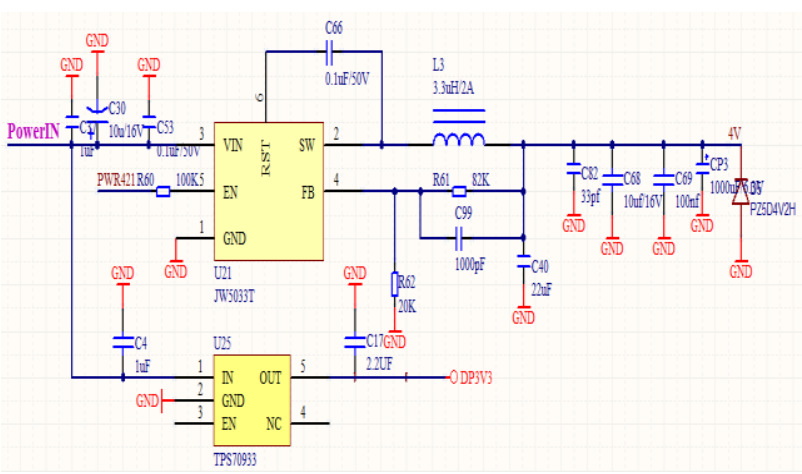

Fig. 11. The circuit diagram of a designed power-supply module used by D/RTUs.



Fig. 12. The PCB layout of the developed MCU module's core board used by D/RTUs.

\section{- Wireless communication module}

Four types of wireless communication modules have been self-developed for use primarily in EMULSION: (1) GPRS; (2) Wi-Fi; (3) NB-IoT; and (4) LTE Cat.1/Cat.4 modules. The GPRS modules utilize MTK 2503 chipsets, the NB-IoT modules MTK 2625 chipsets, the Wi-Fi modules - ESP32 chipsets, and the LTE Cat. 1 and Cat. 4 modules utilize UIS8910DM and MTK 6737 chipsets, respectively. The PCB layout of the developed MTK2503-based GPRS module is shown in Fig. 13.

\section{Conclusion}

This paper has briefly sketched the main design principles of the generic horizontal-type EMULSION IoT platform. More specifically the paper has focused on the heterogenous aspect of the sensor tier of EMULSION, which includes a variety of sensors, monitoring stations, location trackers, actuators, controllers, and guards. Aided by the communication tier, this tier is able to solve associated problems related to the heterogeneity and integration of different IoT things, objects, and devices by utilization of smart communication gateways.

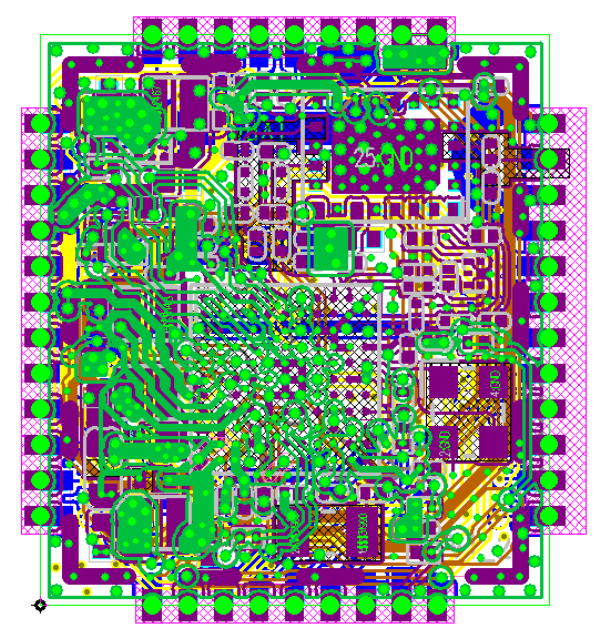

Fig. 13. The PCB layout of the developed MTK2503based GPRS module used by D/RTUs.

Future research work will include further elaboration of novel techniques, algorithms, and models for the effective and efficient service recommendations, the initial results of which are described in [11] [12]. This will be followed by the development of corresponding software (run on multiple mobile platforms) ensuring the best quality of experience (QoE) for consumers when using different IoT services, supplied by EMULSION, primarily in the 'smart health' and 'smart environment control' IoT sectors. The software applications, which are being developed, will be accessible anytime-anywhere-anyhow through any kind of mobile device via the 'best' available wireless access network, in accordance with the $\mathrm{ABC} \& \mathrm{~S}$ communication paradigm.

\section{References:}

[1]. Lueth, K.L., IoT 2021 in review: The 10 Most Relevant IoT Developments of the Year. 2022, IoT Analytics.

[2]. Wopata, M., 5 Things to Know About the IoT Platforms Market. 2021, IoT Analytics.

[3]. Wegner, P., IoT Platform Companies Landscape 2021/2022: Market consolidation has started. 2021, IoT Analytics.

[4]. Ganchev, I., Z. Ji, and M. O'Droma. A Generic Multi-Service Cloud-Based IoT Operational Platform - EMULSION. in 2019 International Conference on Control, Artificial Intelligence, Robotics \& Optimization (ICCAIRO). 2019.

[5]. Ganchev, I., Z. Ji, and M. O'Droma, Designing a Low-Cost Location Tracker for Use in IoT Applications, in XXXIII General Assembly and Scientific Symposium of the International 
Union of Radio Science (URSI GASS 2020). 2020, IEEE. p. x1-x2.

[6]. Ganchev, I., Z. Ji, and O.D. M., Low-cost and ultra-low-power consuming RTUs for use in IoT systems. Int. J. Circ., Syst. and Sig. Process., 2020. 14: p. 76-82.

[7]. Razouk, W., D. Sgandurra, and K. Sakurai, A new security middleware architecture based on fog computing and cloud to support IoT constrained devices, in Proceedings of the 1st International Conference on Internet of Things and Machine Learning. 2017, Association for Computing Machinery: Liverpool, United Kingdom. p. Article 35.

[8]. Andonov, V., S. Poryazov, and E. Saranova, Generalized net representations of control structures in service systems theory. Advanced Studies in Contemporary Mathematics (Kyungshang), 2020. 30(1): p. 49-60.

[9]. Andonov, V., S. Poryazov, and E. Saranova, Generalized net representations of elements of service systems theory. Advanced Studies in Contemporary Mathematics (Kyungshang), 2019. 29(2): p. 179-189.

[10]. Ganchev, I., User-Centric and Context-Aware $A B C \& S$. International Journal "Information Technologies \& Knowledge", 2015. 9(3): p. 203-215.
[11]. Zhou, D., et al., Novel SDDM Rating Prediction Models for Recommendation Systems. IEEE Access, 2021. 9: p. 101197101206.

[12]. Zhang, H., et al., FeatureMF: An Item Feature Enriched Matrix Factorization Model for Item Recommendation. IEEE Access, 2021. 9: p. 65266-65276.

Ivan Ganchev carried out the conceptualization, methodology, project administration and supervision, funding acquisition, writing of the original draft and editing of the final paper.

Zhanlin Ji carried out data curation, formal analysis, investigation, validation, software development, and resource management.

This publication has emanated from joint research conducted with the financial support of the Bulgarian National Science Fund (BNSF) under the Grant No. KP-06-IP-CHINA/1 (КП06-ИП-КИТАЙ/1) and the S\&Т Major Project of the Science and Technology Ministry of China, Grant No. 2017YFE0135700.

\section{Creative Commons Attribution License 4.0 (Attribution 4.0 International, CC BY 4.0)}

This article is published under the terms of the Creative Commons Attribution License 4.0

https://creativecommons.org/licenses/by/4.0/deed.en_US 